Pacific Journal of Mathematics

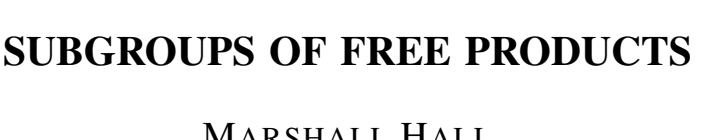




\title{
SUBGROUPS OF FREE PRODUCTS
}

\author{
Marshall Hall, Jr.
}

1. Introduction. It was first shown by Kurosch [3] that a subgroup $H$ of a free product

$$
G=\prod_{\nu}^{*} A_{\nu}
$$

is itself a free product

$$
H=F * \prod_{j}^{*} x_{j}^{-1} U_{j} x_{j}
$$

of a free group $F$ and conjugates $x_{j}^{-1} U_{j} x_{j}$ of subgroups $U_{j}$ of free factors $A$. The original proof of Kurosch involved constructing the free factors of $H$ one at a time, and both the proof and the construction depended on transfinite induction. A later proof by Baer and Levi [1] was topological in nature. More recently a proof has been given by Kuhn [2].

The present paper gives a new proof of this theorem, which, apart from the use of well-ordering, is purely algebraic. It is shorter and simpler than the Kurosch proof. In terms of a semi-alphabetical ordering of $G$, a set $K$ of elements generating $H$ is found, and it is then shown from the properties of $K$ that $H$ is a free product of factors as stated above.

2. The theorem of Kurosch. The result is the following:

TheOrem OF KUROSCH. A subgroup $H \neq 1$ of a free product

$$
G=\prod_{\nu}^{*} A_{\nu}
$$

is itself a free product

Received April 2, 1952.

Pacific J. Math. 3 (1953), 115-120 


$$
H=F * \prod_{j}^{*} x_{j}^{-1} U_{j} x_{j},
$$

where $F$ is a free group and each $x_{j}^{-1} U_{j} x_{j}$ is the conjugate of a subgroup $U_{j}$ of one of the free factors $A_{\nu}$ of $G$.

Proof. The elements of the free factors of $G$ may be well ordered by beginning with the identity, then taking an ordering of the free factors, and within a free factor taking an ordering of the elements $\neq 1$. Based on this ordering we define an alphabetical ordering for the elements of $G$. Write

$$
g=a_{1} a_{2} \cdots a_{t}
$$

as the reduced form of an element $g$ of $G$. The void product is the identity; and for $g \neq 1$ each $a_{i}$ is an element $\neq 1$ of one of the free factors $A_{\nu}$, and no two consecutive terms $a_{i}, a_{i+1}(i=1, \cdots, t-1)$ belong to the same free factor $A_{\nu}$. The length $l(g)$ of an element $g$ is defined as zero for $g=1$, and for $g \neq 1$ as the number $t$ of terms in its reduced form (1). We define the alphabetical ordering of elements by ordering successively on

1) the length of $g$;

2.1) the order of the first term $a_{1}$ if $g=a_{1} a_{2} \ldots a_{t}$ is its reduced form;

2.2) the order of $a_{2}$;

2.t) the order of $a_{t}$.

This is clearly a well ordering of the elements of $G$.

We now define a second ordering for the elements of $G$, the semi-alphabetical ordering. For this we write an element $g$ of even length $t=2 r$ in the form $g=$ $\alpha \beta^{-1}$, where $l(\alpha)=l(\beta)=r$; and an element $g$ of odd length $t=2 s+1$ in the form $g=\alpha a_{s+1} \beta^{-1}$, where $l(\alpha)=l(\beta)=s$. The semi-alphabetical ordering for elements $g$ is determined successively by:

1) the length of $g$;

2) for $g=\alpha \beta^{-1}$ of even length by 2.1) the alphabetical order of $\alpha$, and by 2.2 ) the alphabetical order of $\beta$;

3) for $g=\alpha a_{s+1} \beta^{-1}$ of odd length by 3.1) the alphabetical order of $\alpha$, by 3.2 ) the alphabetical order of $\beta$, and by 3.3) the order of $a_{s+1} \cdot$ 
The proof that the subgroup $H$ of $G$ is a free product will be carried out by selecting, in terms of the semi-alphabetical ordering, a subset $K$ of the elements of $H$ and showing 1 ) that the elements of $K$ generate $H$ and then 2 ) that the elements $K$ generate a free product

$$
F * \prod_{j}^{*} x_{j}^{-1} U_{j} x_{j},
$$

where $F$ is a free group and each $U_{j}$ is a subgroup of some free factor $A$.

The set $K$ of elements shall consist of all elements $k \neq 1$ such that 1 ) $k \in H$, and 2) $k$ does not belong to the group generated by the elements of $H$ which precede $k$ in the semi-alphabetical ordering.

Since $H \neq 1$, the first $h \neq 1$ of $H$ belongs to the set $K$, and so $K$ is not vacuous. Consider the group $[K]$ generated by the set $K$. Clearly $[K] \subseteq H$. If $[K] \neq H$, there must be a first $h \in H$ such that $h \notin[K]$. Such an $h$ does not belong to $K$, and so is a product of elements $h_{i}$ preceding $h$ and belonging to $H$. But these $h_{i}$ belong to $[K]$, and so $h$ as a product of these $h_{i}$ 's also belongs to $[K]$. Hence $[K]=H$, and this covers the first part of the proof.

We shall use the sign < for numerical inequalities and for both the alphabetical and semi-alphabetical orderings. It will be clear from the context which meaning is appropriate, the semi-alphabetical ordering applying to entire words, the alphabetical to beginnings or endings of words. Writing $u \neq 1$ in the form $u=\alpha \beta^{-1}$ or $u=\alpha a \beta^{-1}$, we cannot have $\beta=\alpha$ for words of even length since $\alpha \alpha^{-1}=1$. For elements of odd length, $\beta=\alpha$ is possible; and those elements of $H$ of the form $\alpha a \alpha^{-1}$ for fixed $\alpha$, and $a^{\prime}$ s belonging to some fixed $A_{\nu}$, together with the identity, form a subgroup $\alpha B \alpha^{-1}$ conjugate to $B \subseteq A_{\nu}$. Let us call elements $\alpha a \alpha^{-1}$ transforms. Let us extend the set $K$ to a larger set $T$ which consists of $K$ and, for each $\alpha$ and $A_{\nu}$, those transforms $\alpha a^{\prime} \alpha^{-1}, a^{\prime} \in A_{\nu}$, generated by transforms $\alpha a \alpha^{-1}, a \in A_{\nu}$, belonging to $K$. Hence $T$ consists of elements of $H$ not generated by their predecessors and transforms $\alpha a^{\prime} \alpha^{-1}$ generated by earlier transforms of the same kind.

An element $h \in H$ can be written in the form

$$
h=u_{1} u_{2} \cdots u_{t} \text {, }
$$

where $u_{i} \in T$ or $T^{-1}$ (the set of inverses of elements in $T$ ). Moreover we can take (2) so that a) $u_{i} u_{i+1} \neq 1(i=1, \ldots, t-1)$ and $\left.\mathrm{b}\right)$ no two consecutive $u_{i}, u_{i+1}$ belong to the same conjugate group $\alpha B \alpha^{-1}, B \subseteq A_{\nu}$. If these conditions are satisfied then we shall say that $u_{1} \cdots u_{t}$ is in half-reduced form. 
The theorem will follow immediately if it can be shown that any nonvacuous half-reduced form cannot be the identity. For then it will follow that the elements of $K$ which are not transforms generate a free group $F$, and that $H$ is the free product of $F$ and the conjugates $a B \alpha^{-1}, B \subseteq A_{\nu}$.

If $u$ is an element of $K$, and $u^{-1} \neq u$, then $u<u^{-1}$ since $u=\left(u^{-1}\right)^{-1}$ and $u^{-1}$ cannot be a predecessor of $u$. Also if $u \neq v$ are elements of $K$, then $w=$ $u^{\epsilon} v^{\eta} \quad(\epsilon, \eta= \pm 1)$ will follow both $u$ and $v$, since any two of $u, v, w$ generate the third and by the choice of $K$ neither $u$ nor $v$ is generated by predecessors. These two principles are the main tools in studying the way in which the elements of $T$ and $T^{-1}$ combine. In reducing a product $a_{1} a_{2} \cdots a_{m}$ in $G$, where each $a_{i}$ belongs to one of the free factors, we say that $a_{i}$ and $a_{i+1}$ amalgamate into $a_{i}^{\prime}$ if $a_{i}$ and $a_{i+1}$ belong to the same free factor $A$ and $a_{i} a_{i+1}=a_{i}^{\prime}$, and that they cancel if $a_{i} a_{i+1}=1$.

\section{LeMmA 1. If $u=\alpha \beta^{-1}$ or $\alpha a \beta^{-1} \in T$, and $\beta \neq \alpha$, then $\alpha<\beta$.}

Proof. Since $\beta \neq \alpha$, we have $u \in K$; and if $\beta<\alpha$, we would have $u^{-1}<u$. Thus the elements of $T$ are of three kinds:

1) $l(u)$ even, $u=\alpha \beta^{-1}, \alpha<\beta, u \in K$;

2) $l(u)$ odd, $u=\alpha a \beta^{-1}, \alpha<\beta, u \in K$;

3 ) $l(u)$ odd, $u=\alpha a \alpha^{-1}$, generated by transforms of the same kind in $K$.

LEMMA 2. If $u \neq v$ belong to $T$ and are not both in the same conjugate $\alpha B \alpha^{-1}$, and $w$ is any one of $u^{\epsilon} v^{\eta}$ or $v^{\eta} u^{\epsilon}(\epsilon, \eta= \pm 1)$, then $w$ follows both $u$ and $v$ in the semi-alphabetical ordering. This leads to the following restrictions on cancellation and amalgamation in the product $w$ :

1) If $u=\alpha \beta^{-1}, \beta$ does not cancel, and if $\alpha$ cancels, then the adjacent term of $\beta^{-1}$ does not amalgamate;

2) if $u=\alpha a \beta^{-1}, \alpha<\beta, \alpha$ and $a$ do not cancel, and if $\beta$ cancels, then $a$ does not amalgamate;

3) if $u=\alpha a \alpha^{-1} \in \alpha B \alpha^{-1}, \alpha$ and $a$ do not cancel, and if $v^{n}=\alpha a^{\prime} \sigma$, with $a, a^{\prime} \in A_{\nu}$, then $a^{\prime}$ is the earliest element in the coset $B a^{\prime}$.

Proof. Of the two different elements $u$ and $v$ belonging to $T$, let the letter $u$ represent the earlier, so that $u<v$. If $w$ does not follow both $u$ and $v$, then $w<v$. Here the possibility $w=v$ may be eliminated at once since it would imply $u=1$, which cannot hold, or $u=v^{2}$ or $v^{-2}$. Now the square of a transform $v$ is 1 or is a similarly transform, while if $v$ is not a transform then $l\left(v^{2}\right)>l(v)$. 
In either case $u=v^{2}$ or $v^{-2}$ is impossible.

Since any two of $u, v, w$ generate the third, $w$ must be the third if $v$ belongs to $K$. Thus we need only consider cases with $v=\alpha a \alpha^{-1} \in \alpha B \alpha^{-1}$ a transform. Now with $u<v$, since $u \notin \alpha B \alpha^{-1}$, we also have $u<v^{*}$, where $v^{*}$ is any transform in $\alpha B \alpha^{-1}$. Hence if the canceling between $u$ and $v=\alpha a \alpha^{-1}$ involves only $\alpha$ (or $\alpha^{-1}$ ), the same will hold for $u$ and some $v^{*}=\alpha a^{*} \alpha^{-1} \in K$, yielding a product $w^{*}=u^{\epsilon} v^{*}$ or $v^{*} u^{\epsilon}$ with $w^{*}<v^{*}$ contrary to $v^{*} \in K$. Hence the canceling between $u$ and $v=\alpha a \alpha^{-1}$ involves all of $\alpha$ and cancellation or amalgamation with the center term $a$. Thus $u^{\epsilon}=\alpha a^{\prime \prime} \sigma^{-1}$, where $a^{\prime \prime}$ amalgamates or cancels with $a$. Since $u<v=\alpha a \alpha^{-1}$, either $l(\sigma)<l(\alpha)$ or $l(\sigma)=l(\alpha)$; and $u=\sigma a^{\prime \prime-1} \alpha^{-1}$, with $\sigma<\alpha$. In either event, $u$ and $\sigma\left(a^{\prime \prime-1} a^{*} a^{\prime \prime}\right) \sigma^{-1}$ precede and generate a $v^{*}=\alpha a^{*} \alpha^{-1} \in K$, a contradiction. Thus in all cases we reach a contraction if $w \leq v$, and so $w$ follows both $u$ and $v$.

In consequence of the fact that all eight products $u^{\epsilon} v^{\eta}$ and $v^{\eta} u^{\epsilon}$ follow both $u$ and $v$, we have the restrictions on canceling and amalgamating listed in the theorem. These say explicitly that not more than half of either $u$ or $v$ cancels, and that in cases where canceling and amalgamating with one replaces an initial (or final) segment of the other with another segment of the same length, the result is an element later in the ordering.

LeMmA 3. In a product $u_{1} u_{2} \cdots u_{t}$ with $u_{i} \in T \cup T^{-1} \quad(i=1, \cdots, t)$, $u_{i} u_{i+1} \neq 1(i=1, \cdots, t-1)$, and $u_{i}, u_{i+1}$ not both in the same group $\alpha B \alpha^{-1}$ $\left(B \subseteq A_{\nu}\right)$, the reduced form will end as follows:
1) $\beta^{-1} \quad$ if $u_{t}=\alpha \beta^{-1}$,
2) $b^{*} \alpha^{-1}$ if $u_{t}=\left(\alpha \beta^{-1}\right)^{-1}$,
3) $a^{*} \beta^{-1}$ if $u_{t}=\alpha a \beta^{-1}, \alpha<\beta$,
4) $a^{-1} \alpha^{-1}$ if $u_{t}=\left(\alpha a \beta^{-1}\right)^{-1}, \alpha<\beta$,
5) $a^{*} \alpha^{-1}$ if $u_{t}=\alpha a \alpha^{-1}$.

Here $b^{*}$ in 2) and $a^{*}$ in 5), are either the term immediately preceding in $u_{t}$ or are amalgamations with a similar term in $u_{t-1}$. In 3$)$, a* can involve amalgamation with $u_{t-1}$ and $u_{t-2}$.

Proof. This lemma will be proved by induction on $t$, being trivial for $t=1$. For $t=2$, the results come directly from Lemma 2 with the added observation that for $u=\alpha \beta^{-1}$ or $\alpha a \beta^{-1}$ the cancellation in $u^{2}$ does not go through $\alpha$ or $\beta$. 
In proving the induction from $t$ to $t+1$, we need only apply Lemma 2 to each of the five cases listed above, as well as each of the five possibilities for $u_{t+1}$, using only one additional property not an immediate consequence of Lemma 2. This is as follows: It may happen that $u_{t}=\alpha a \alpha^{-1}$, that $\alpha$ cancels, and that $a$ amalgamates with $u_{t-1}=\sigma a^{1-1} \alpha^{-1}$, and similarly with $u_{t+1}=\alpha a^{\prime \prime} \lambda$. Now by Lemma 2 each of $a^{\prime}$ and $a^{\prime \prime}$ is the earliest element in its own coset $B a^{\prime}, B a^{\prime \prime}$. If $a^{\prime-1} a a^{\prime \prime}=1$ this would mean that $a^{\prime}$ and $a^{\prime \prime}$ were in the same coset, and so $a^{\prime \prime}=a^{\prime \prime}, a=1, u_{t}=1$, a contradiction. Hence $a^{\prime-1} a a^{\prime \prime} \neq 1$, and the reduced form of $u_{t-1} u_{t} u_{t+1}$ is $\sigma\left(a^{\prime-1} a a^{\prime \prime}\right) \lambda$. This is the only way in which amalgamation can involve as many as three consecutive terms in any product $u_{1} u_{2} \cdots u_{m}$ which is half reduced.

In establishing the ending of the reduced form for the half reduced expression $h=u_{1} u_{2} \cdots u_{t}$, we have shown $\dot{a}$ fortiori that $h \neq 1$, and hence that $H$ is the free product of the infinite cyclic groups generated by the elements $\alpha \beta^{-1}$ and $\alpha a \beta^{-1}(a<\beta)$ and the conjugates $\alpha B \alpha^{-1}$ of subgroups $B$ of free factors $A$.

\section{REFERENCES}

1. R. Baer and F. Levi, Freie Produkte und ihre Untergruppen, Compositio Math. 3 (1936), 391 - 398.

2. H. W. Kuhn, Subgroup theorems for groups presented by generators and relations, Ann. of Math. 56 (1952), 22-46.

3. A. Kurosch, Die Untergruppen der freien Produkte von beliebigen Gruppen, Math. Ann. 109 (1934), 647-660.

The Ohio State University 


\section{PACIFIC JOURNAL OF MATHEMATICS}

\section{EDITORS}

\author{
R. M. Foeinson \\ University of California \\ Berkeley 4, California \\ E. HEWITt \\ University of Washington \\ Seattle 5, Washington
}

R. P. DILWOR TH

California Institute of Technology

Pasadena 4, California

E. F. BECKENBACH

University of California

Los Angeles 24, California

\section{ASSOCIATE EDITORS}

$\begin{array}{llll}\text { H. BUSEMANN } & \text { P. R. HALMOS } & \text { BøRGE JESSEN } & \text { J. J. STOKER } \\ \text { HERBERT FFDERER } & \text { HEINZ HOPF } & \text { PAUL LÉVY } & \text { E. G. STR AUS } \\ \text { MARSHALL IIALI } & \text { R. D. JAMES } & \text { GEORGE PÓLYA } & \text { KỎSAKU YOSIDA }\end{array}$

\section{SPONSORS}

UNIVERSITY OF BRITISH COLUMBIA CAIIFORNIA INSTITUTE OF TECHNOLOGY UNIVERSITY OF CALIFORNIA, BERKELEY UNIVERSITY OF CAILIFORNIA, DAVIS UNIVERSITY OF CALIFORNIA, LOS ANGELES UNIVERSITY OF CALIFORNIA, SANTA BARBARA UNIVERSITY OF NEVADA OREGON STATE COLLEGE UNIVERSITY OF OREGON
UNIVERSITY OF SOUTHERN CALIFORNIA STANFORD RESEARCH INSTITUTE STANFORD UNIVERSITY WASHINGTON STATE COLLEGE UNIVERSITY OF WASHINGTON

AMERICAN MATHEMATICAL SOCIETY NATIONAL BUREAU OF STANDARDS, INSTITUTE FOR NUMERICAL ANALYSIS

$$
\begin{gathered}
\text { Vari-Type Composition by } \\
\text { Elaine Barth } \\
\text { Delores Wierman } \\
\text { With the cooperation of } \\
\text { E. F. Beckenbach } \\
\text { E. G. Straus }
\end{gathered}
$$

Printed in the United States of America by Edwards Brothers, Inc., Ann Arbor, Michigan 


\section{Pacific Journal of Mathematics}

\section{Vol. 3, No. 1 \\ March, 1953}

Herbert Busemann, Volume in terms of concurrent cross-sections ......... 1

L. Carlitz, Some special equations in a finite field ................. 13

Homer V. Craig and Billie Braden Townsend, On certain metric

extensors ....................................... 25

Philip J. Davis and Henry Pollak, Linear functionals and analytic

continuation problems ............................. 47

Jacob C. E. Dekker, The constructivity of maximal dual ideals in certain

Boolean algebras ................................. 73

Harley M. Flanders, The norm function of an algebraic field extension .... 103

Marshall Hall, Subgroups of free products . . . . . . . . . . . . . . . . . 115

Israel (Yitzchak) Nathan Herstein, Finite multiplicative subgroups in

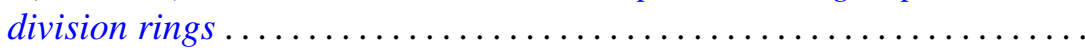

Joseph Lawson Hodges, Jr. and Murray Rosenblatt, Recurrence-time moments in random walks ............................ 127

Alfred Horn, The normal completion of a subset of a complete lattice and lattices of continuous functions ........................ 137

Fulton Koehler, Estimates for the errors in the Rayleigh-Ritz method...... 153

M. H. Martin, The Monge-Ampère partial differential equation

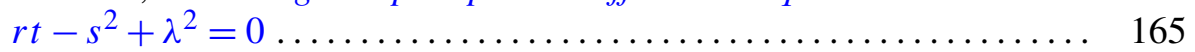

John E. Maxfield, Normal k-tuples ......................... 189

Jack E. McLaughlin, Structured theorems for relatively complemented lattices............................................ 197

William H. Mills, A system of quadratic Diophantine equations ......... 209

T. S. Motzkin, Ernst Gabor Straus and F. A. Valentine, The number of farthest points ................................. 221

G. Power, Forces on the boundary of a dielectric ............... 233

Ralph Gordon Selfridge, Approximations with least maximum error....... 247 